\title{
The Spectral Relation between the Cube-Connected Cycles and the Shuffle-Exchange Network
}

\author{
Christian Riess, Volker Strehl, Rolf Wanka \\ Department of Computer Science, University of Erlangen-Nuremberg, Germany \\ \{christian.riess, strehl, rwanka\}@informatik.uni-erlangen.de
}

\begin{abstract}
We investigate the relation between the spectral sets (i. e., the sets of eigenvalues, disregarding multiplicities) of two $d$-dimensional networks popular in parallel computing: the Cube-Connected Cycles network $\mathrm{CCC}(d)$ and the Shuffle-Exchange network $\mathrm{SE}(d)$. We completely characterize their spectral sets. Additionally, it turns out that for any odd $d$, the $\operatorname{SE}(d)$-eigenvalues set is precisely the same as the $\operatorname{CCC}(d)$ eigenvalues set. For any even $d$, however, the $\mathrm{SE}(d)$-eigenvalues form a proper subset of the set of $\operatorname{CCC}(d)$-eigenvalues.
\end{abstract}

\section{Introduction}

Background. Popular Hypercubic networks used as parallel machines are the Butterfly network, the Cube-Connected Cycles network, the Shuffle-Exchange network and the DeBruijn network. For a collection of their properties and many algorithms for them, see, e. g., [Lei92]. In particular, these constant-degree networks are able to execute so-called normal hypercube algorithms with only constant slowdown if compared to the execution time on the hypercube which has non-constant degree ([Lei92]).

Among the characteristic parameters of networks, the eigenvalues of their adjacency matrices are very important (e.g., see [CDS95, Chu97, BK05] for comprehensive studies). They reflect many structural properties of the network. For instance, from the eigenvalues it can immediately be decided whether the network is bipartite (see Proposition 4 below). Expansion properties, bisection problems, the mixing time of Markov chains and the computation of the isoperimetric number [DT98, Bül97] are fields of application of eigenvalues in algorithmic graph theory.

In the area of parallel computing, there is a direct connection between the eigenvalues and the routing number [ACG94]. Further applications can be found in the analysis of parallel load-balancing algorithms [RSW98] and in the design of interconnection networks [EKM03].

The set of eigenvalues is called the spectral set. In the spectrum of a graph, additionally the multiplicities of the eigenvalues are considered. For formal definitions, see Subsec. 2.2. Previously, only the full spectral sets of the DeBruijn network [DT98] and the two variants of the Butterfly network [EKM03, Sch01] have been known. 
New results. In this paper, we exactly characterize the spectral sets of the Cube-Connected Cycles $\operatorname{CCC}(d)$ and the Shuffle-Exchange network $\operatorname{SE}(d)$ in terms of the spectra of cycles with self-loops that have weights from $\{-1,+1\}$ (see Theorems 1 and 2).

It turns out (see Theorem 3) that for any odd $d$ the set of the $\mathrm{SE}(d)$-eigenvalues is precisely the same as the set of the $\operatorname{CCC}(d)$-eigenvalues. For any even $d$, however, the $\operatorname{SE}(d)$ eigenvalues form a proper subset of the set of $\operatorname{CCC}(d)$-eigenvalues. The odd case is particularly remarkable because the networks differ in the number of vertices by a factor of $d$, and hence, the eigenvalues have different multiplicities. Also, there is no obvious way of identifying the eigenvalues bijectively. In fact, corresponding eigenvalues can only be found on scattered cycles of the networks, and a new argument on the involved eigenspaces is necessary in order to find all $\operatorname{CCC}(d)$-eigenvalues in the eigenvalue set of $\operatorname{SE}(d)$, if $d$ is odd. For an instructive (counter-)example for $d=6$, see Sec. 6 .

If $d$ is even, -3 that is an eigenvalue of $\operatorname{CCC}(d)$ is not an eigenvalue of $\operatorname{SE}(d)$. In fact, when $d$ becomes larger, the size of the difference set increases. Let $\Delta_{d}=\mid \operatorname{SpS}(\operatorname{CCC}(d)) \backslash$ $\operatorname{SpS}(\operatorname{SE}(d)) \mid$ denote the number of eigenvalues of $\operatorname{CCC}(d)$ that are not eigenvalues of $\operatorname{SE}(d)$. In terms of $\Delta_{d}$, the result of this paper can be stated as: if $d$ is odd, $\Delta_{d}=0$, and $\Delta_{d} \geq 1$, if $d$ is even. Explicit computation shows for even $d, d \leq 20$ :

\begin{tabular}{r|rrrrrrrrr}
$d$ & 4 & 6 & 8 & 10 & 12 & 14 & 16 & 18 & 20 \\
\hline$\Delta_{d}$ & 1 & 3 & 1 & 9 & 7 & 42 & 21 & 179 & 160
\end{tabular}

Known results. We briefly mention some known spectral sets, denoted by $\operatorname{SpS}($.$) :$

Let $L\left(n_{1}, \ldots, n_{d}\right)$ denote the $d$-dimensional $n_{1} \times \cdots \times n_{d}$-array. Then

$$
\operatorname{SpS}\left(L\left(n_{1}, \ldots, n_{d}\right)\right)=\left\{2 \sum_{i=1}^{d} \cos \left(\frac{\pi j_{i}}{n_{i}+1}\right) \mid 1 \leq j_{i} \leq n_{i} \text { for } i \in\{1, \ldots, d\}\right\} .
$$

In the following, $L_{n}:=L(n)$ denotes the linear array of length $n$.

Let $\Theta\left(n_{1}, \ldots, n_{d}\right)$ denote the $d$-dimensional $n_{1} \times \cdots \times n_{d}$-torus. Then

$$
\operatorname{SpS}\left(\Theta\left(n_{1}, \ldots, n_{d}\right)\right)=\left\{2 \sum_{i=1}^{d} \cos \left(\frac{2 \pi j_{i}}{n_{i}}\right) \mid 0 \leq j_{i} \leq n_{i}-1 \text { for } i \in\{1, \ldots, d\}\right\} .
$$

As the adjacency matrices of tori are block-circulant there is a comparatively simple way to compute their spectra (see Proposition 1 in Subsec. 2.2). In the following, $C_{n}:=\Theta(n)$ denotes the cycle of length $n$.

Interestingly, the spectral sets of other popular networks can be expressed in terms of linear arrays $L_{n}$ and cycles $C_{n}$ (in the following, $a \cdot M_{k}$ denotes the product of the adjacency matrix $M_{k}$ and the scalar $a$ ). For the $d$-dimensional Butterfly network $\operatorname{BF}(d)$ (for proofs, see [Sch01, EKM03]),

$$
\operatorname{SpS}(\operatorname{BF}(d))=\bigcup_{k=0}^{d+1} \operatorname{SpS}\left(2 L_{k}\right) .
$$


Similarly, for the Butterfly network with wrap-around edges [Sch01, EKM03, CFGM03], $\operatorname{SpS}(\mathrm{W}-\mathrm{BF}(d))=\operatorname{SpS}\left(2 C_{d}\right) \cup \bigcup_{k=0}^{d} \operatorname{SpS}\left(2 L_{k}\right)$. Let $\mathrm{DB}(D, d)$ denote the $D$-ary $d$-dimensional DeBruijn graph. Then (for a proof, see [DT98]),

$$
\operatorname{SpS}(\operatorname{DB}(D, d))=\operatorname{SpS}\left(D \cdot C_{1}\right) \cup \bigcup_{\tau=1}^{d} \operatorname{SpS}\left(D \cdot L_{\tau}\right) .
$$

Organization of paper. The paper is organized as follows: In the next section, we define the networks to be investigated, give the necessary definitions regarding graph spectra, and state important properties. In Sections 3 and 4, we exactly characterize the spectra of the Cube-Connected Cycles network and the Shuffle-Exchange network. In Sec. 5, we prove that, if $d$ is odd, the sets of eigenvalues are identical, whereas, if $d$ is even, the set of eigenvalues of $\operatorname{SE}(d)$ is a proper subset of the set of eigenvalues of $\operatorname{CCC}(d)$. That there is no simple correspondence between the eigenvalues of $\operatorname{CCC}(d)$ and $\operatorname{SE}(d$ is exemplified in Sec. 6.

\section{Preliminaries}

In this section, we introduce the Cube-Connected Cycles network and the Shuffle-Exchange network. We present some of their properties, present tools for computing their eigenvalues, and introduce some necessary notations.

\section{1 $\operatorname{CCC}(d), \operatorname{SE}(d)$, and Their Properties}

The $d$-dimensional Cube-Connected Cycles network $\operatorname{CCC}(d)$ has been introduced by Preparata and Vuillemin in [PV81]. It is the undirected graph with vertex set $V=\{(j, \mathbf{a}) \mid$ $\left.1 \leq j \leq d, \mathbf{a} \in\{0,1\}^{d}\right\}$ and edge set $E=\{\{(j, \mathbf{a}),((j \bmod d+1, \mathbf{a})\} \mid 1 \leq j \leq d, \mathbf{a} \in$ $\left.\{0,1\}^{d}\right\} \cup\left\{\{(j, \mathbf{a}),(j, \mathbf{a}[j])\} \mid 1 \leq j \leq d, \mathbf{a}=\left(a_{d}, \ldots, a_{j}, \ldots, a_{1}\right) \in\{0,1\}^{d}, \mathbf{a}[j]=\right.$ $\left.\left(a_{d}, \ldots, 1-a_{j}, \ldots, a_{1}\right)\right\}$. $\mathrm{CCC}(d)$ has $d \cdot 2^{d}$ vertices and is 3-regular.

The $d$-dimensional Shuffle-Exchange network $\operatorname{SE}(d)$ has been introduced by Stone [Sto71]. It is the undirected graph with vertex set $V=\{0,1\}^{d}$ and edge set $E=\{\{\mathbf{a}, \mathbf{a}[1]\} \mid \mathbf{a} \in$ $\left.\{0,1\}^{d}\right\} \cup\left\{\{\mathbf{a}, \operatorname{cyc}(\mathbf{a})\} \mid \mathbf{a} \in\{0,1\}^{d}, \operatorname{cyc}\left(a_{d}, \ldots, a_{2}, a_{1}\right)=\left(a_{1}, a_{d}, \ldots, a_{2}\right)\right\}$. The edges of the first subset are called exchange edges, the edges of the second subset are called shuffle edges. Here, we also consider multiple shuffle edges such that $\operatorname{SE}(d)$ is also 3 -regular.

$\mathrm{CCC}(3)$ and $\operatorname{SE}(3)$ are shown in Fig. 1 and 2, resp. Note the self-loops at vertices 000 and 111 of $\mathrm{SE}(3)$ that ensure $\mathrm{SE}(d)$ being 3-regular.

Note that for $d$ being even, $\operatorname{CCC}(d)$ is bipartite [LPS ${ }^{+} 98$ ]. The cycles of $\operatorname{CCC}(d)$ are characterized directly by the corresponding sequence $\mathbf{a}$.

Cycles in $\operatorname{SE}(d)$ are more complex to describe. Let $S$ be a set of integers. Let $\mathbf{a}=$ 
$\left(a_{k}, \ldots, a_{1}\right) \in S^{k}$. a is an aperiodic $S$-sequence if there is no $t>1$ and $\mathbf{b}$ with $\mathbf{a}=\mathbf{b}^{t}$. $\mathbf{a}$ is a Lyndon $S$-sequence [CFL58] if it is an aperiodic $S$-sequence and the lexicographically smallest under all sequences obtained by cyclically shifting $\mathbf{a}$.

The shuffle edges of $\mathrm{SE}(d)$ form disjoint shuffle cycles. Every cycle is uniquely characterized by a Lyndon $\{0,1\}$-sequence. In this paper, using the correspondence $0 \mapsto+1$ and $1 \mapsto-1$, we shall say that every shuffle cycle is characterized by a different Lyndon $\{-1,+1\}$-sequence and that every possible Lyndon $\{-1,+1\}$-sequence of length $k$ with $k$ being a divisor of $d$ characterizes a different shuffle cycle.

\subsection{Eigenvalues, Spectral Sets, and Computation Tools}

Let $A$ be the adjacency matrix of an undirected graph $G=(V, E)$ (with multiple edges allowed; the entry $a_{i j}$ is the number of edges between nodes $i$ and $j$ ). In the rest of this paper, we identify $G$ and $A$. Let $n=|V|$ denote the number of vertices, and let $I_{n}$ denote the $n \times n$ unit matrix. Then the polynomial $\chi(A ; z)=\operatorname{det}\left(z \cdot I_{n}-A\right)$ is the characteristic polynomial of $G$, and the set $\operatorname{SpS}(G)=\{\lambda \mid \chi(A ; \lambda)=0\}$ is the spectral set of roots of $\chi(A ; z)$. Such a root is called eigenvalue. In this paper, we do not consider the multiplicities of the eigenvalues.

Let $A$ and $B$ be two matrices. The Kronecker product $A \otimes B$ is the matrix one obtains from $A$ by replacing entry $a_{i j}$ by $a_{i j} \cdot B$.

A $(q \cdot p) \times(q \cdot p)$ matrix $B$ is called $(p, q)$-block circulant iff there are $p \times p$ matrices $B_{1}, \ldots, B_{q}$ such that

$$
B=\left(\begin{array}{cccc}
B_{1} & B_{2} & \cdots & B_{q} \\
B_{q} & B_{1} & \cdots & B_{q-1} \\
\vdots & \vdots & \ddots & \vdots \\
B_{2} & B_{3} & \cdots & B_{1}
\end{array}\right)=:\left\langle B_{1}, B_{2}, \cdots, B_{q}\right\rangle
$$

If $p=1, B$ is called circulant. Block circulant matrices are well studied (e. g., see [Dav79]). In particular, there is a nice way to determine $\chi(B ; z)$ and to compute $\operatorname{SpS}(B)$. Let $\omega_{q}=\mathrm{e}^{2 \pi \mathrm{i} / q}=\cos (2 \pi / q)+\mathrm{i} \cdot \sin (2 \pi / q)$ be a primitive $q$-th root of unity. Let $B(x)=$ $\sum_{k=1}^{q} x^{k-1} \cdot B_{k}$. The following proposition on the characteristic polynomial and the spectral set of block circulant matrices is very useful for the computation of the spectral sets of neatly constructed graphs.

Proposition 1 ([Dav79]) Let $B=\left\langle B_{1}, \ldots, B_{q}\right\rangle$ be a $(p, q)$-block circulant matrix. Then

$$
\chi(B ; z)=\prod_{j=0}^{q-1} \chi\left(B\left(\omega_{q}^{j}\right) ; z\right)=\prod_{j=0}^{q-1} \chi\left(\sum_{k=1}^{q} \omega_{q}^{j \cdot(k-1)} \cdot B_{k} ; z\right) .
$$

For the spectral set, this means

$$
\operatorname{SpS}(B)=\bigcup_{j=0}^{q-1} \operatorname{SpS}\left(\sum_{k=1}^{q} \omega_{q}^{j \cdot(k-1)} \cdot B_{k}\right)
$$


E. g., as the $n$-cycle $C_{n}$ is $(1, n)$-block circulant with $C_{n}=\langle 0,1,0 \ldots, 0,1\rangle, \operatorname{SpS}\left(C_{n}\right)=$ $\left\{\omega_{n}^{j}+\omega_{n}^{(n-1) j} \mid 0 \leq j \leq n-1\right\}=\{2 \cos (2 \pi j / n) \mid 0 \leq j \leq n-1\}$. Similarly, the spectra of $d$-dimensional tori can be computed in this way resulting in the spectral set mentioned in Sec. 1.

Proposition 1 can be used directly to prove the following useful observation.

Proposition 2 Let $G$ and $X$ be $p \times p$ square matrices. For the $(p, 2)$-block circulant matrix $\langle G, X\rangle$, we have $\chi(\langle G, X\rangle ; z)=\chi(G+X ; z) \cdot \chi(G-X ; z)$.

Proposition 3 Let $B=\left\langle B_{1}, \ldots, B_{q}\right\rangle$ be a real $(p, q)$-block circulant matrix with $B_{q-j+1}=B_{j}^{\mathrm{T}}, 1 \leq j \leq q$. Then the following holds.

(a) $B\left(\omega_{q}^{-j}\right)=B\left(\omega_{q}^{j}\right)^{\mathrm{T}}, B\left(\omega_{q}^{j}\right)$ is self-adjoint, $1 \leq j \leq q$.

(b) $\chi\left(B\left(\omega_{q}^{-j}\right) ; z\right)=\chi\left(B\left(\omega_{q}^{j}\right)^{\mathrm{T}} ; z\right)$.

(c) If $q$ is odd, then there is a polynomial $g(z)$ such that $\chi(B ; z)=\chi(B(1) ; z) \cdot g(z)^{2}$

(d) If $q$ is even, then there is a polynomial $g(z)$ such that

$$
\chi(B ; z)=\chi(B(1) ; z) \cdot \chi(B(-1) ; z) \cdot g(z)^{2}
$$

(e) By (c) and (d), all eigenvalues of $B$ that do not come from $B(1)$ and $B(-1)$ occur in pairs and belong to two-dimensional eigenspaces.

For the proof of Proposition 3, (a) and (b) can be shown directly, and for (c) and (d), use Proposition 1, (b) and that $\omega_{q}^{-j}=\omega_{q}^{q-j}$, for all $j$.

The following well known facts will be essential for the proof that the spectral sets of $\mathrm{CCC}(d)$ and $\mathrm{SE}(d)$ are different if $d$ is even.

Proposition 4 Let $G$ be a connected graph with maximal degree $\Delta$.

(a) [Bol98, p. 263] $G$ is regular iff $\Delta \in \operatorname{SpS}(G)$.

(b) [Bol98, p. 263] If $-\Delta \in \operatorname{SpS}(G)$, then $G$ is regular and bipartite.

(c) [BK05, p. 379] $G$ is bipartite iff for all $\lambda \in \operatorname{SpS}(G)$, also $-\lambda \in \operatorname{SpS}(G)$.

\subsection{Further Notation}

For a sequence $\mathbf{s}=\left(s_{k}, \ldots, s_{1}\right) \in \mathbb{Z}^{k}$, and $k \leq n$, let $D_{n}[\mathbf{s}]$ be the $n \times n$-diagonal matrix with $s_{1}, \ldots, s_{k}, 0, \ldots, 0$ in the main diagonal. In particular, $I_{n}=D_{n}\left[1^{n}\right]$ is the identity matrix.

$C_{n}=\langle 0,1,0, \ldots, 0,1\rangle$ denotes the circulant adjacency matrix of the cycle of length $n$. For a sequence $\mathbf{s}=\left(s_{k}, \ldots, s_{1}\right) \in \mathbb{Z}^{k}$, and $k \leq n, C_{n}[\mathbf{s}]=C_{n}+D_{n}[\mathbf{s}]$. For reasons of 
consistency, we need a special definition for the cases $n=1$ and $n=2: C_{1}[s]=(2+s)$ and $C_{2}\left[s_{2}, s_{1}\right]=\left(\begin{array}{cc}s_{1} & 2 \\ 2 & s_{2}\end{array}\right)$.

$L_{n}=\left(l_{i j}\right)$ denotes the $n \times n$-adjacency matrix of the linear array of length $n$. It is identical to $C_{n}$ except for the entries $l_{1 n}=l_{n 1}=0$ (instead of being 1). $L_{n}[\mathbf{s}]$ is defined analogously to $C_{n}[\mathbf{s}]$.

\section{The Spectral Set of $\operatorname{CCC}(d)$}

In order to compute the spectral set of $\operatorname{CCC}(d)$, we generalize the notion of cube-connectedness.

Let $d$ be a non-negative integer, and let $G$ be a graph with $n, n \geq d$, nodes, numbered from 1 through $n$. The $d$-dimensional Cube-Connected $G$-network is the graph $\operatorname{CC}(G, d)$ with vertex set $\{1, \ldots, n\} \times\{0,1\}^{d}$. Two nodes $(i, \mathbf{a})$ and $(j, \mathbf{a})$ are adjacent iff $i$ and $j$ are adjacent in $G$. Furthermore, two nodes $(i, \mathbf{a})$ and $(i, \mathbf{b})$ are adjacent iff $\mathbf{a}$ and $\mathbf{b}$ differ exactly at the $i$ th bit. So, $\operatorname{CC}(G, d)$ consists of $2^{d}$ copies of $G$ that are interconnected in a hypercubic way. Using the length- $d$ cycle $C_{d}$ as $G$, we have with $\operatorname{CC}\left(C_{d}, d\right)$ the famous Cube-Connected Cycles network.

Let $\mathbf{s}=\left(s_{d}, \ldots, s_{1}\right) \in\{-1,+1\}^{d}$. The graph $G[\mathbf{s}]$ is obtained from $G$ by adding a self-loop with weight $s_{i}$ to node $i$, for all $i \in\{1, \ldots, d\}$.

Theorem 1 Let $G$ be a graph with $n, n \geq d$, nodes. Then

$$
\chi(\mathrm{CC}(G, d) ; z)=\prod_{\boldsymbol{s} \in\{-1,+1\}^{d}} \chi(G[\boldsymbol{s}] ; z) .
$$

Proof. Let $R_{n, d}$ be the $n \times n$ matrix with all entries being 0 except for $r_{d d}$ which is 1 , and let $X_{d-1}=I_{2^{d-1}} \otimes R_{n, d}$. Then the adjacency matrix of $\mathrm{CC}(G, d)$ can be expressed as follows:

$$
\mathrm{CC}(G, d)=\left(\begin{array}{cc}
\mathrm{CC}(G, d-1) & X_{d-1} \\
X_{d-1} & \mathrm{CC}(G, d-1)
\end{array}\right)
$$

By Proposition 2, this means that the characteristic polynomial of the whole graph can be expressed as follows:

$$
\begin{aligned}
\chi(\mathrm{CC}(G, d) ; z) & =\chi\left(\mathrm{CC}(G, d-1)+X_{d-1} ; z\right) \cdot \chi\left(\mathrm{CC}(G, d-1)-X_{d-1} ; z\right) \\
& =\chi\left(\mathrm{CC}\left(G\left[0^{d-1}, 1\right], d-1\right) ; z\right) \cdot \chi\left(\mathrm{CC}\left(G\left[0^{d-1},-1\right], d-1\right) ; z\right) \\
& =\prod_{\mathbf{s} \in\{-1,+1\}^{d-1}} \chi(G[\mathbf{s}, 1] ; z) \cdot \prod_{\mathbf{s} \in\{-1,+1\}^{d-1}} \operatorname{SpS}(G[\mathbf{s},-1] ; z)
\end{aligned}
$$

For (1), note that $\mathrm{CC}(G, d-1) \pm X_{d-1}$ is a copy of $\mathrm{CC}(G, d-1)$ where all nodes $(d, \mathbf{a})$ get a self-loop added with weight $-1,+1$. (2) follows by induction. $\square$ (Theorem 1 )

By choosing $G$ being the $d$-cycle $C_{d}$, we obtain: 
Corollary 1 For $\mathrm{CCC}(d)$,

$$
\begin{aligned}
\chi(\mathrm{CCC}(d) ; z) & =\prod_{\boldsymbol{s} \in\{-1,+1\}^{d}} \chi\left(C_{d}[\boldsymbol{s}] ; z\right) \\
\operatorname{SpS}(\operatorname{CCC}(d)) & =\bigcup_{\boldsymbol{s} \in\{-1,+1\}^{d}} \operatorname{SpS}\left(C_{d}[\boldsymbol{s}]\right) .
\end{aligned}
$$

Hence, the spectral set of $\mathrm{CCC}(d)$ is exactly the union of the spectral sets of all $d$-cycles where the nodes of the cycles are weighted with all possible $\{-1,+1\}$-sequences.

The application of Eq. (1) from the proof of Theorem 1 can be interpreted as editing the original graph. The resulting graph has exactly the same spectrum as the original graph. Fig. 1 shows the corresponding graphs for $d=3,2,1$, when $\operatorname{CCC}(3)$ is edited. In the end, the are the 3 -cycles with weighted self-loops. In the light of the editing, we call them residual cycles.
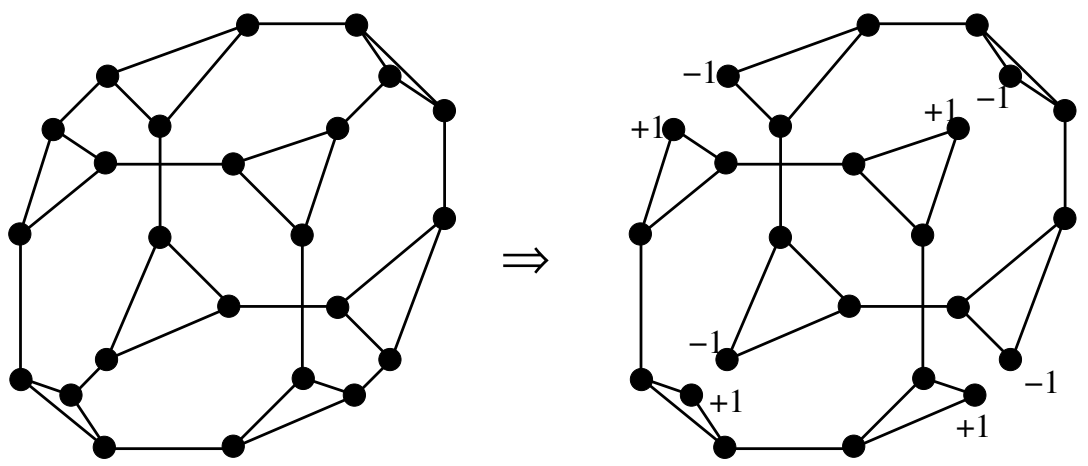

$\Downarrow$
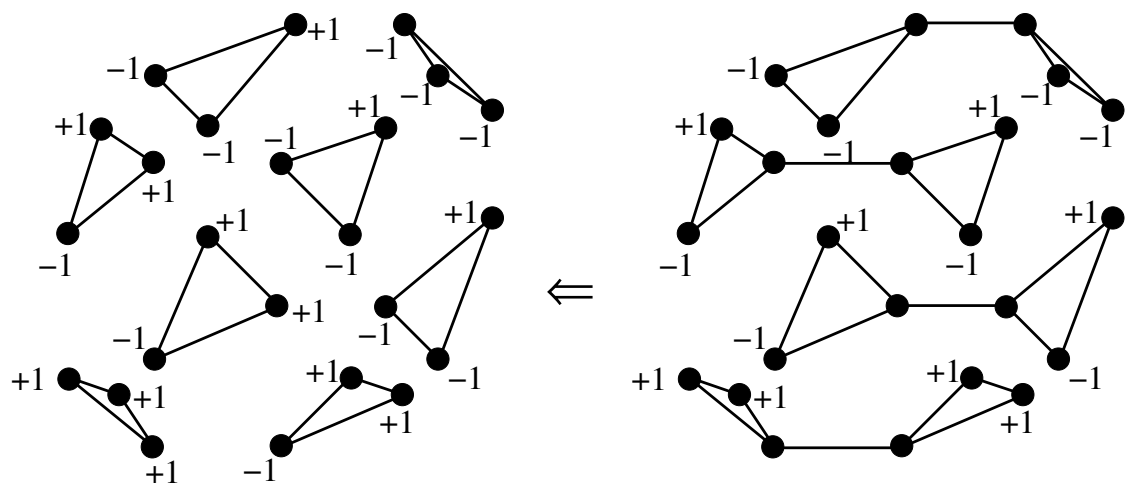

Figure 1: Editing the Cube-Connected Cycles network CCC(3).

Similarly, the spectrum of the Cube-Connected Lines network [Par86] can be characterized 
in terms of linear arrays $L_{d}$.

\section{The Spectral Set of SE $(d)$}

In order to obtain the adjacency matrix of $\mathrm{SE}(d)$, we describe the shuffle edges and the exchange edges separately, i. e., $\operatorname{SE}(d)=\operatorname{Sh}(d)+\operatorname{Ex}(d)$.

Lemma 1 (a) Let $U(d)=\left(\begin{array}{l}1 \\ 0\end{array}\right) \otimes I_{2^{d-1}} \otimes\left(\begin{array}{ll}1 & 0\end{array}\right)+\left(\begin{array}{l}0 \\ 1\end{array}\right) \otimes I_{2^{d-1}} \otimes\left(\begin{array}{ll}0 & 1\end{array}\right)$.

Then, $\operatorname{Sh}(d)=U(d)+U(d)^{\mathrm{T}}$.

(b) $\operatorname{Ex}(d)=I_{2^{d-1}} \otimes\left(\begin{array}{ll}0 & 1 \\ 1 & 0\end{array}\right)$

In order to prove Lemma 1 , it suffices to identify the binary address a of a node with the number $(\mathbf{a})_{2}+1$.

Let $H_{d}=\frac{1}{2^{d / 2}} \cdot\left(\begin{array}{cc}1 & 1 \\ 1 & -1\end{array}\right)^{\otimes d}$, where $A^{\otimes d}$ denotes $\overbrace{A \otimes \cdots \otimes A}^{d} . H_{d}$ is the well-known Hadamard matrix. Note that $H_{d}^{-1}=H_{d}$.

Lemma 2 (a) $H_{d}^{-1} \cdot \operatorname{Sh}(d) \cdot H_{d}=\operatorname{Sh}(d)$

(b) $H_{d}^{-1} \cdot \operatorname{Ex}(d) \cdot H_{d}=I_{2^{d-1}} \otimes\left(\begin{array}{cc}1 & 0 \\ 0 & -1\end{array}\right)$.

For the proof, (b) can be shown easily by induction on $d$.

In order to show (a), a simple, but tedious computation shows that $H_{d}$ commutes with both $U(d)$ and $U(d)^{\mathrm{T}}$, hence with $\operatorname{Sh}(d)$.

Theorem 2 For the Shuffle-Exchange network $\mathrm{SE}(d)$,

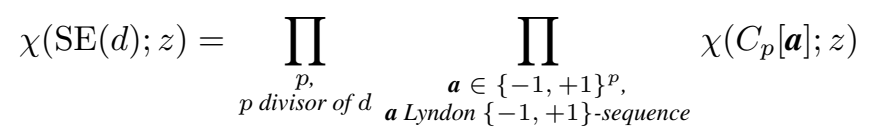

Proof. We have $\chi(\mathrm{SE}(d) ; z)=\chi\left(H_{d}^{-1} \cdot \mathrm{SE}(d) \cdot H_{d} ; z\right)$. By Lemma 2 ,

$$
\chi(\mathrm{SE}(d) ; z)=\chi\left(\mathrm{Sh}(d)+I_{2^{d-1}} \otimes\left(\begin{array}{cc}
1 & 0 \\
0 & -1
\end{array}\right) ; z\right)
$$

So the shuffle cycles are left unchanged, the exchange edges disappear, and all nodes get an additional $-1,+1$-self-loop, according to their binary addresses. Every shuffle cycle is characterized by Lyndon $\{0,1\}$-sequences. Now the weights of the vertices of the 
shuffle cycles are characterized by the respective Lyndon $\{-1,+1\}$-sequence, where 0 s are replaced with +1 and 1 s with -1 .

Recall the special definition of $C_{1}[a]$ and $C_{2}[a, b]$.

$\square$ (Theorem 2)

As the computation of $H_{d}^{-1} \cdot \mathrm{SE}(d) \cdot H_{d}$ does not change the characteristic polynomial, this computation can again be regarded as editing the original graph. This time, the exchange edges are removed and their nodes receive an additional self-loop with weights $-1,+1$. The result of editing $\mathrm{SE}(3)$ is shown in Fig. 2 Again, we call the obtained cycles residual cycles.

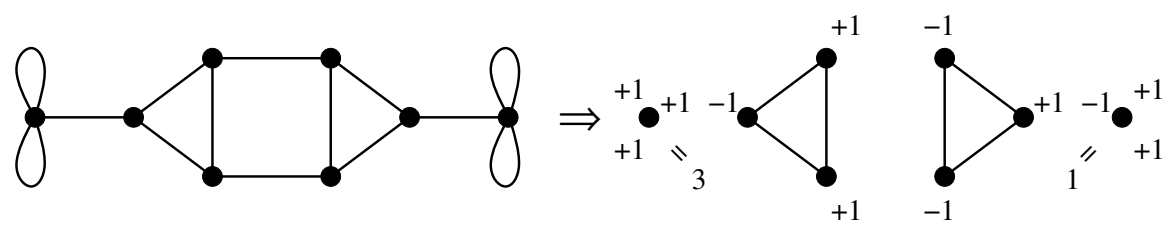

Figure 2: Result of editing the Shuffle Exchange network SE(3).

\section{Spectral Relation between CCC and SE}

The following Theorem shows the (surprisingly close) relation between the spectral set of $\mathrm{CCC}(d)$ and $\mathrm{SE}(d)$ :

Theorem 3 The spectral sets of the d-dimensional Cube-Connected Cycles network and the Shuffle-Exchange network are equal if and only if $d$ is odd. Otherwise, the spectral set of the Shuffle-Exchange network is a proper subset of the spectral set of the CubeConnected Cycles network.

More formally, the following properties hold:

1. $\quad \operatorname{SpS}(\operatorname{SE}(d)) \subseteq \operatorname{SpS}(\operatorname{CCC}(d))$

2. $d$ odd: $\operatorname{SpS}(\operatorname{SE}(d))=\operatorname{SpS}(\mathrm{CCC}(d))$

3. $d$ even: $\operatorname{SpS}(\mathrm{SE}(d)) \subsetneq \operatorname{SpS}(\mathrm{CCC}(d))$, since $-3 \in \mathrm{SpS}(\mathrm{CCC}(d)) \backslash \mathrm{SpS}(\mathrm{SE}(d))$

The rest of this section is devoted to the proof of Theorem 3. We start with the first case:

\section{Proof of Theorem 3, part 1:}

Take any factor $\chi\left(C_{p}[\mathbf{a}] ; z\right)$ from $\chi(\mathrm{SE}(d) ; z)$, according to Theorem 2 . There are two cases to be distinguished: If $p=d$, then $\chi\left(C_{p}[\mathbf{a}] ; z\right)=\chi\left(C_{d}[\mathbf{a}] ; z\right)$. Thus, by Corollary 1 , $\chi\left(C_{p}[\mathbf{a}] ; z\right)$ is a factor of $\chi(\operatorname{CCC}(d) ; z)$. Consider now the case, that $p<d$. Let $d=$ $p \cdot q$ and let $\delta_{i j}$ be the Kronecker delta, i. e., $\delta_{i j}=1$ if $i=j$, and $\delta_{i j}=0$ if $i \neq j$. Using the block circulant structure of $C_{d}\left[\mathbf{a}^{q}\right]$, it can be seen that $\chi\left(C_{p}[\mathbf{a}] ; z\right)$ is a factor of $\chi\left(C_{d}\left[\mathbf{a}^{q}\right] ; z\right)$ : 
$C_{d}\left[\mathbf{a}^{q}\right]=\left\langle A_{0}, A_{1}, \ldots, A_{q-1}\right\rangle$ is a block circulant matrix with $A_{0}=L_{p}[\mathbf{a}]$, $A_{1}=\left[\delta_{x, p} \delta_{y, 1}\right]_{1 \leq x, y \leq p}, A_{q-1}=A_{1}^{\mathrm{T}}$ and $A_{2}=\cdots=A_{q-2}=(0)$, such that Propositions 2 and 3 are satisfied. One can see that $\chi\left(C_{p}[\mathbf{a} ; z]\right)$ is a factor of $\chi\left(C_{d}\left[\mathbf{a}^{q}\right] ; z\right)$ by noting from Proposition 1 that

$$
\chi\left(C_{d}\left[\mathbf{a}^{q}\right] ; z\right)=\prod_{j=0}^{q-1} \chi\left(A_{0}+\omega_{q}^{j} A_{1}+\omega_{q}^{-j} A_{q-1} ; z\right),
$$

hence $\chi\left(C_{p}[\mathbf{a} ; z]\right)=\chi\left(A_{0}+\omega_{q}^{0} \cdot A_{1}+\omega_{q}^{0} A_{q-1} ; z\right)$ is also a factor of $\chi(\operatorname{CCC}(d) ; z)$.

\section{Proof of Theorem 3, part 2:}

We already know that for all $d$, $\operatorname{SpS}(\operatorname{SE}(d)) \subseteq \operatorname{SpS}(\operatorname{CCC}(d))$. Thus, for odd $d$, it remains to be shown that $\operatorname{SpS}(\operatorname{SE}(d)) \supseteq \operatorname{SpS}(\operatorname{CCC}(d))$. Take any factor $\chi\left(C_{d}[\mathbf{b}] ; z\right)$ from $\chi(\mathrm{CCC}(d) ; z)$, there are again two cases:

If $\mathbf{b} \in\{-1,+1\}^{d}$ is aperiodic, then $\chi\left(C_{d}[\mathbf{b}] ; z\right)$ is a factor of $\chi(\operatorname{SE}(d) ; z)$, since $\mathbf{b}$ is already minimal with respect to periodicity, as presented in Theorem 2 . If $\mathbf{b} \in\{-1,+1\}^{d}$ is periodic, then $\mathbf{b}=\mathbf{a}^{q}$ for some aperiodic $\mathbf{a} \in\{-1,+1\}^{p}$ with $d=p \cdot q$. Using Proposition 3, we know that $\chi\left(C_{d}[\mathbf{b}] ; z\right)=\chi\left(C_{d}[\mathbf{a}] ; z\right) \cdot g(z)^{2}$. Note that the corresponding eigenspace of the double eigenvalues that result from $g(z)^{2}$ is two-dimensional.

A sequence $\mathbf{b}^{\prime} \in\{-1,+1\}^{d}$ that differs from $\mathbf{b}$ in one single (arbitrary) position - w.l.o.g. in the first position - is always aperiodic, thus $\chi\left(C_{d}(\mathbf{b}) ; z\right)$ is a factor of $\chi(\operatorname{SE}(d) ; z)$. We show that the characteristic polynomial factors as

$$
\chi\left(C_{d}\left[\mathbf{b}^{\prime}\right] ; z\right)=g(z) \cdot h(z),
$$

which proves this part of the Theorem:

Take any of the two-dimensional eigenspaces of $C_{d}[\mathbf{b}]$ belonging to some root $\lambda$ of $g(z)$. The vectors which have 0 in their first component form a one-dimensional (at least) subspace of eigenvectors of $C_{d}\left[\mathbf{b}^{\prime}\right]$ for the same eigenvalue $\lambda$, thus all these eigenvalues $\lambda$ are preserved in $\chi\left(C_{d}\left[\mathbf{b}^{\prime}\right] ; z\right)$ with multiplicity of at least 1.

Note that because of Proposition 3(b) and (c) the above eigenspace argument only holds if $d$ is odd. A counterexample for $d=6$ is presented in Sec. 6 .

\section{Proof of Theorem 3, part 3:}

Let $d$ be even. $\operatorname{CCC}(d)$ is 3-regular and bipartite [LPS +98 ]. So by Proposition 4, $-3 \in$ $\operatorname{SpS}(\operatorname{CCC}(d))$.

If $d$ is not a power of 2, then $\operatorname{SE}(d)$ contains cycles of odd length. Hence, $\operatorname{SE}(d)$ is not bipartite, so by Proposition $4,-3 \notin \operatorname{SpS}(\operatorname{SE}(d))$. Even if $d$ is a power of 2 , then $\operatorname{SE}(d)$ at least contains self-loops at the nodes $0^{d}$ and $1^{d}$, so here $\mathrm{SE}(d)$ is not bipartite, which means that $-3 \notin \operatorname{SpS}(\mathrm{SE}(d))$.

Recall that -3 is not the only eigenvalue of $\operatorname{CCC}(d), d$ even and $d \in\{6,10,12,14 \ldots\}$, not occurring in the spectral set of $\operatorname{SE}(d)$ (see the remark on the number of different eigenvalues in Sec. 1 and specifically the example in the next section). 


\section{An Instructive Example on the Eigenvalues of $\operatorname{CCC}(6)$}

Here we demonstrate by an example that there might be no simple correspondence between the eigenvalues of $\mathrm{CCC}(d)$ and $\operatorname{SE}(d)$, and that the eigenspace argument used in the proof of Theorem 3 might be necessary.

Corollary 1 and Theorem 2 state that the spectra of $\operatorname{CCC}(d)$ and $\operatorname{SE}(d)$ consist of the spectra of cycles where the vertices have self-loops with weights from $\{-1,+1\}$. As $\mathrm{SE}(d)$ consists of cycles of different sizes, there is no direct correspondence between the CCC-eigenvalues and the SE-eigenvalues.

For example, consider the case $d=6$, and edit $\operatorname{CCC}(6)$ and $\operatorname{SE}(6)$ in order to get the residual cycles with self-loops from $\{-1,+1\}$.

For $\operatorname{CCC}(6)$, the residual cycle $C_{6}[-1,+1,-1,+1,-1,+1]$ which corresponds to the periodic binary sequence $010101=(01)^{3}$ has the characteristic polynomial $\left(z^{2}-2\right)^{2}\left(z^{2}-\right.$ 5).

For $\mathrm{SE}(6)$, the residual cycle $C_{2}[-1,+1]$ which corresponds to the non-periodic binary sequence 01 has the characteristic polynomial $z^{2}-5$. So it is at this moment not yet clear whether the roots $\pm \sqrt{2}$ of $z^{2}-2$ originating, among others, from the CCC-cycle 010101 are eigenvalues of $\mathrm{SE}(6)$. In this case, they are because the characteristic polynomial of $C_{6}[-1,+1,-1,+1,+1,+1,+1$,$] which corresponds to the non-periodic binary sequence$ 000101 is $\left(z^{2}-2\right)\left(z^{4}-2 z^{3}-5 z^{2}+8 z+2\right)$.

On the other hand, for $\operatorname{CCC}(6)$, the residual cycle $C_{6}[-1,-1,+1,-1,-1,+1]$ which corresponds to the binary sequence 011011 has the characteristic polynomial $z(z-2)(z+$ $1)(z+2)\left(z^{2}+z-4\right)$. The factor $z^{2}+z-4$ does not occur in any characteristic polynomial of the residual cycles of $\mathrm{SE}(6)$, so its roots $-\frac{1}{2} \pm \frac{1}{2} \sqrt{17}$ are not eigenvalues of $\mathrm{SE}(6)$, but only of $\mathrm{CCC}(6)$.

\section{Conclusion}

In this paper, we completely characterized the spectral sets of $\operatorname{CCC}(d)$ and $\operatorname{SE}(d)$ (Theorems 1 and 2, resp.). In order to compute the eigenvalues, we used a "graph editing" technique that illustrates the computation process.

Curiously, it turns out (Theorem 3) that the eigenvalue sets are identical if $d$ is odd. If $d$ is even, the set of eigenvalues of $\operatorname{SE}(d)$ is a proper subset of the set of eigenvalues of $\mathrm{CCC}(d)$. In order to show this result, we had to use the corresponding eigenspaces because there is no simple correspondence between the cycles of the residual graphs. 


\section{References}

[ACG94] Noga Alon, Fan R. K. Chung, and Ronald L. Graham. Routing permutations on graphs via matchings. SIAM Journal on Discrete Mathematics, 7:513-530, 1994.

[BK05] Andreas Baltz and Lasse Kliemann. Spectral Analysis. In Ulrik Brandes and Thomas Erlebach, editors, Network Analysis, pages 373-417. Springer, 2005.

[Bol98] Béla Bollobás. Modern Graph Theory. Springer, New York, 1998.

[Bü197] J. Bültermann. A New Upper Bound for the Isoperimetric Number of deBruijn Networks. Applied Mathematics Letters, 10:97-100, 1997.

[CDS95] D. M. Cvetković, M. Doob, and H. Sachs. Spectra of Graphs. J. A. Barth Verlag, Heidelberg, 3rd edition, 1995.

[CFGM03] Francesc Comellas, Miguel Angel Fiol, Joan Gimbert, and Margarida Mitjana. The spectra of wrapped butterfly digraphs. Networks, 42:15-19, 2003.

[CFL58] K. T. Chen, R. H. Fox, and R. C. Lyndon. Free Differential Calculus, IV. The Quotient Groups of the Lower Central Series. The Annals of Mathematics, Second Series, 68(1):81-95, 1958.

[Chu97] Fan R. K. Chung. Spectral Graph Theory. American Mathematical Society, 1997.

[Dav79] Philip J. Davis. Circulant Matrices. Wiley, New York, 1979.

[DT98] Charles Delorme and Jean-Pierre Tillich. The spectrum of de Bruijn and Kautz graphs. European Journal of Combinatorics, 19(3):307-319, 1998.

[EKM03] Robert Elsässer, Rastislav Královič, and Burkhard Monien. Sparse topologies with small spectrum size. Theoretical Computer Science, 307:549-565, 2003.

[Lei92] F. Thomson Leighton. Introduction to Parallel Algorithms and Architectures: Arrays, Trees, Hypercubes. Morgan Kaufmann Publishers, San Mateo, CA, 1992.

$\left[\mathrm{LPS}^{+}\right.$98] K. Li, Y. Pan, H. Shen, G. H. Young, and S. Q. Zheng. Lower Bounds for Dynamic Tree Embedding in Bipartite Networks. Journal of Parallel and Distributed Computing, 53:119-143, 1998.

[Par86] Ian Parberry. On recurrent and recursive interconnection patterns. Information Processing Letters, 22:285-289, 1986.

[PV81] Franco Preparata and Jean Vuillemin. The Cube-Connected Cycles: a versatile network for parallel computation. Communications of the ACM, 24:300-309, 1981.

[RSW98] Yuval Rabani, Alistair Sinclair, and Rolf Wanka. Local Divergence of Markov chains and the analysis of iterative load-balancing schemes. In Proc. 39th IEEE Foundations of Computer Science (FOCS), pages 694-703, 1998.

[Sch01] Gunnar Schmidt. Über die Spektren wichtiger Graphklassen. Studienarbeit, Universität Paderborn, 2001.

[Sto71] H. S. Stone. Parallel Processing with the Perfect Shuffle. IEEE Transactions on Computers, C-20:153-161, 1971. 